\title{
Fractal Intersections and Products via Algorithmic Dimension
}

\author{
Neil Lutz* \\ Department of Computer and Information Science, University of Pennsylvania, \\ Philadelphia, PA, USA \\ neillutz@gmail.com
}

\begin{abstract}
Algorithmic dimensions quantify the algorithmic information density of individual points and may be defined in terms of Kolmogorov complexity. This work uses these dimensions to bound the classical Hausdorff and packing dimensions of intersections and Cartesian products of fractals in Euclidean spaces. This approach shows that a known intersection formula for Borel sets holds for arbitrary sets, and it significantly simplifies the proof of a known product formula. Both of these formulas are prominent, fundamental results in fractal geometry that are taught in typical undergraduate courses on the subject.
\end{abstract}

1998 ACM Subject Classification F.1.3 Complexity Measures and Classes

Keywords and phrases algorithmic randomness, geometric measure theory, Hausdorff dimension, Kolmogorov complexity

Digital Object Identifier 10.4230/LIPIcs.MFCS.2017.58

\section{Introduction}

Classical fractal dimensions, among which Hausdorff dimension [12] is the most important, refine notions of measure to quantitatively classify sets of measure 0. In 2000, J. Lutz [15] showed that Hausdorff dimension can be simply characterized using betting strategies called gales, and that this characterization can be effectivized in order to quantitatively classify non-random infinite data objects. This effective Hausdorff dimension and other, related algorithmic dimensions have been applied to multiple areas of computer science and have proven especially useful in algorithmic information theory [25].

The connection between algorithmic and classical dimensions has more recently been exploited in the other direction, i.e., to apply algorithmic information theoretic methods and intuition to classical fractal geometry (e.g., [29, 2]). A point-to-set principle of J. Lutz and N. Lutz [16], stated here as Theorem 6, characterizes the classical Hausdorff dimension of any set in $\mathbb{R}^{n}$ in terms of the algorithmic dimensions of its individual points.

In the same work, J. Lutz and N. Lutz showed that the point-to-set principle gives rise to a new, pointwise technique for dimensional lower bounds, and, as a proof of concept, used this technique to give an algorithmic information theoretic proof of Davies's 1971 [7] theorem stating that every Kakeya set in $\mathbb{R}^{2}$ has Hausdorff dimension 2. This bounding technique has since been used by N. Lutz and Stull [18] to make new progress on a problem in classical fractal geometry by deriving an improved lower bound on the Hausdorff dimension of generalized Furstenberg sets, as defined by Molter and Rela [26].

\footnotetext{
* This research was done at Rutgers University and supported in part by National Science Foundation Grant 1445755 .
} 

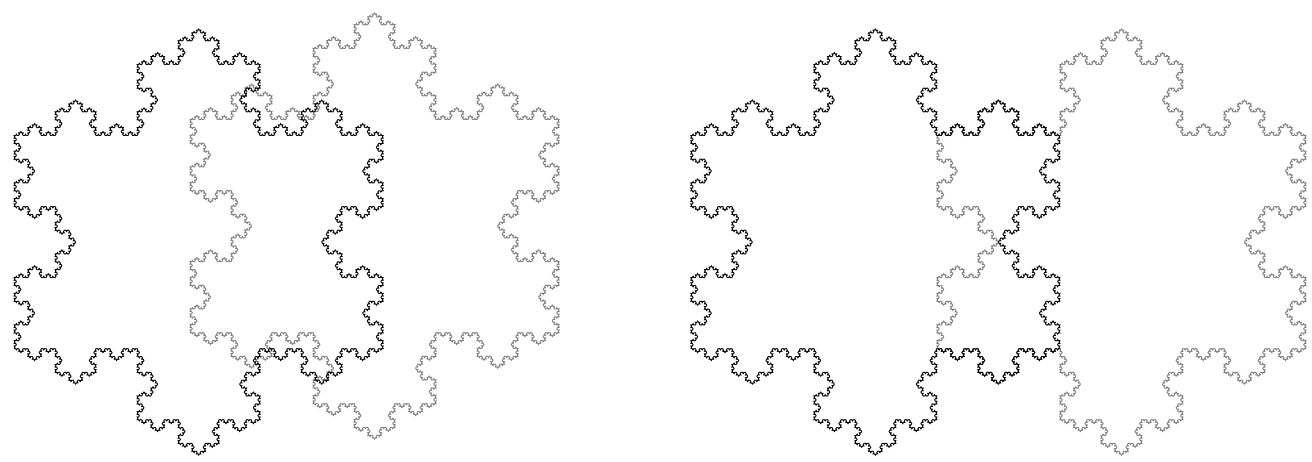

Figure 1 Let $E$ and $F$ both be Koch snowflakes, which have Hausdorff dimension $\log _{3} 4 \approx 1.26$. Left: Theorem 1 states that, for almost all translation parameters $z \in \mathbb{R}^{2}$, the Hausdorff dimension of the intersection $E \cap(F+z)$ is at most $2 \log _{3} 4-2 \approx 0.52$. Right: For a measure zero set of translations, the intersection may have Hausdorff dimension as large as that of the original sets. Note that Koch curves are Borel sets, so the new generality introduced by Theorem 1 is not required for this example.

The same algorithmic dimensional technique is applied here to bound the dimensions of intersections and products of fractals. Most significantly, we extend the following intersection formula, previously shown to hold when $E$ and $F$ are Borel sets [11], to arbitrary sets $E$ and $F{ }^{1}$ This formula is illustrated in Figure 1.

- Theorem 1. For all $E, F \subseteq \mathbb{R}^{n}$, and for (Lebesgue) almost all $z \in \mathbb{R}^{n}$,

$$
\operatorname{dim}_{H}(E \cap(F+z)) \leq \max \left\{0, \operatorname{dim}_{H}(E \times F)-n\right\},
$$

where $F+z=\{x+z: x \in F\}$.

Our approach also yields a simplified proof of the following known product formula for general sets.

- Theorem 2 (Marstrand [19]). For all $E \subseteq \mathbb{R}^{m}$ and $F \subseteq \mathbb{R}^{n}$,

$$
\operatorname{dim}_{H}(E)+\operatorname{dim}_{H}(F) \leq \operatorname{dim}_{H}(E \times F) .
$$

We use symmetric arguments to derive the known corresponding statements about packing dimension [37, 10], a formulation of fractal dimension that was developed independently by Tricot [37] and Sullivan [36] and is dual to Hausdorff dimension. These results are included here to showcase the versatility of this technique and its ability to capture the exact duality between Hausdorff and packing dimensions.

\section{Classical Fractal Dimensions}

We begin by stating classical, measure-theoretic definitions of the two most well-studied notions of fractal dimension, Hausdorff dimension and packing dimension. These definitions are included here for completeness but are not used directly in the remainder of this work; we will instead apply equivalent characterizations in terms of algorithmic information, as described in Section 3.

1 This result is closely related to the Marstrand Slicing Theorem, as stated in the excellent recent book by Bishop and Peres [4]. The proof given there assumes that a set is Borel, but this assumption was inadvertently omitted from the theorem statement [3]. 
- Definition 2.1 (Hausdorff [12]). For $E \subseteq \mathbb{R}^{n}$, let $\mathcal{U}_{\delta}(E)$ be the collection of all countable covers of $E$ by sets of positive diameter at most $\delta$, where the diameter of any set $U \subseteq \mathbb{R}^{n}$ is given by

$$
\operatorname{diam}(U)=\sup _{x, y \in U}|x-y| .
$$

For all $s \geq 0$, let

$$
H_{\delta}^{s}(E)=\inf \left\{\sum_{i \in \mathbb{N}} \operatorname{diam}\left(U_{i}\right)^{s}:\left\{U_{i}\right\}_{i \in \mathbb{N}} \in \mathcal{U}_{\delta}(E)\right\} .
$$

The $s$-dimensional Hausdorff (outer) measure of $E$ is

$$
H^{s}(E)=\lim _{\delta \rightarrow 0^{+}} H_{\delta}^{s}(E),
$$

and the Hausdorff dimension of $E$ is

$$
\operatorname{dim}_{H}(E)=\inf \left\{s>0: H^{s}(E)=0\right\}=\sup \left\{s: H^{s}(E)=\infty\right\} .
$$

Three desirable properties have made $\operatorname{dim}_{H}$ the most standard notion of fractal dimension since it was introduction by Hausdorff in 1919. First, it is defined on every set in $\mathbb{R}^{n}$. Second, it is monotone: if $E \subseteq F$, then $\operatorname{dim}_{H}(E) \leq \operatorname{dim}_{H}(F)$. Third, it is countably stable: if $E=\bigcup_{i \in \mathbb{N}} E_{i}$, then $\operatorname{dim}_{H}(E)=\sup _{i \in \mathbb{N}} \operatorname{dim}_{H}\left(E_{i}\right)$. These three properties also hold for packing dimension, which was defined much later, independently by Tricot [37] and by Sullivan [36].

- Definition 2.2 (Tricot [37], Sullivan [36]). For all $x \in \mathbb{R}^{n}$ and $\rho>0$, let $B_{\rho}(x)$ denote the open ball of radius $\rho$ and center $x$. For all $E \subseteq \mathbb{R}^{n}$, let $\mathcal{V}_{\delta}(E)$ be the class of all countable collections of pairwise disjoint open balls with centers in $E$ and diameters at most $\delta$. That is, for every $i \in \mathbb{N}$, we have $V_{i}=B_{\rho_{i}}\left(x_{i}\right)$ for some $x_{i} \in E$ and $\rho_{i} \in[0, \delta / 2]$, and for every $j \neq i$, $V_{i} \cap V_{j}=\emptyset$. For all $s \geq 0$, define

$$
P_{\delta}^{s}(E)=\sup \left\{\sum_{i \in \mathbb{N}} \operatorname{diam}\left(V_{i}\right)^{s}:\left\{V_{i}\right\}_{i \in \mathbb{N}} \in \mathcal{V}_{\delta}(E)\right\},
$$

and let

$$
P_{0}^{s}(E)=\lim _{\delta \rightarrow 0^{+}} P_{\delta}^{s}(E) .
$$

The s-dimensional packing (outer) measure of $E$ is

$$
P^{s}(E)=\inf \left\{\sum_{i \in \mathbb{N}} P_{0}^{s}\left(E_{i}\right): E \subseteq \bigcup_{i \in \mathbb{N}} E_{i}\right\},
$$

and the packing dimension of $E$ is

$$
\operatorname{dim}_{P}(E)=\inf \left\{s: P^{s}(E)=0\right\}=\sup \left\{s>0: P^{s}(E)=\infty\right\} .
$$

Notice that defining packing dimension in this way requires an extra step of optimization compared to Hausdorff dimension. More properties and details about classical fractal dimensions may be found in standard references such as [23, 11, 35]. 


\section{$3 \quad$ Algorithmic Fractal Dimensions}

This section defines the effective Hausdorff and packing dimensions in terms of algorithmic information, i.e., Kolmogorov complexity. We also define conditional dimensions and discuss some properties of these dimensions, including their relationships to classical Hausdorff and packing dimensions.

\subsection{Kolmogorov Complexity}

Kolmogorov complexity quantifies the incompressibility of finite data objects. It is most often defined in the space $\{0,1\}^{*}$ of binary strings, but it is readily extended to other discrete domains. For the purposes of this work, the complexity of rational points is most relevant. Hence, fix some standard binary encoding for $n$-tuples of rationals. The Kolmogorov complexity of $p$ is the length of the shortest binary program that outputs $p$. Formally, it is

$$
K(p)=\min _{\pi \in\{0,1\}^{*}}\{|\pi|: U(\pi)=p\}
$$

where $U$ is a fixed universal prefix-free Turing machine and $|\pi|$ is the length of $\pi$. This quantity is also called the algorithmic information content of $p$. The conditional Kolmogorov complexity of $p$ given $q \in \mathbb{Q}^{n}$ is the length of the shortest binary program that outputs $p$ when given $q$ as an input:

$$
K(p \mid q)=\min _{\pi \in\{0,1\}^{*}}\{|\pi|: U(\pi, q)=p\} .
$$

The algorithmic mutual information between $p \in \mathbb{Q}^{m}$ and $q \in \mathbb{Q}^{n}$ measures, informally, the amount that knowledge of $q$ helps in the task of compressing $p$. Formally, it is

$$
I(p: q)=K(p)-K(p \mid q) .
$$

The quantities $K(p), K(p \mid q)$, and $I(p: q)$ may be considered algorithmic versions of entropy $H(X)$, conditional entropy $H(X \mid Y)$, and mutual information $I(X ; Y)$, from classical (Shannon) information theory. See references $[14,27,8]$ for more details on algorithmic information and the connections between algorithmic and classical theories of information.

\subsection{Effective Dimensions}

Using approximation by rationals, Kolmogorov complexity may be further extended to Euclidean spaces [17]. For every $E \subseteq \mathbb{R}^{n}$, define

$$
K(E)=\min \left\{K(p): p \in E \cap \mathbb{Q}^{n}\right\},
$$

where the minimum is understood to be infinite if $E \cap \mathbb{Q}^{n}$ is empty. This is the length of the shortest program that outputs some rational point in E. The Kolmogorov complexity of $x \in \mathbb{R}^{n}$ at precision $r \in \mathbb{N}$ is given by

$$
K_{r}(x)=K\left(B_{2^{-r}}(x)\right)
$$

the length of the shortest program that outputs any precision-r rational approximation of $x$. $K_{r}(x)$ may also be described as the algorithmic information content of $x$ at precision $r$, and similarly, $K_{r}(x) / r$ is the algorithmic information density of $x$ at precision $r$. This ratio does not necessarily converge as $r \rightarrow \infty$, but it does have limiting bounds in $[0, n]$. These limits are used to define effective dimensions. 
- Definition $3.1([15,24,1,17])$. Let $x \in \mathbb{R}^{n}$.

1. The effective Hausdorff dimension of $x$ is

$$
\operatorname{dim}(x)=\liminf _{r \rightarrow \infty} \frac{K_{r}(x)}{r} .
$$

2. The effective packing dimension of $x$ is

$$
\operatorname{Dim}(x)=\limsup _{r \rightarrow \infty} \frac{K_{r}(x)}{r} .
$$

These dimensions were originally defined by J. Lutz [15] and Athreya, Hitchcock, J. Lutz, and Mayordomo [1], respectively. The original definitions were in Cantor space and used gales, which are betting strategies that generalize martingales, emphasizing the unpredictability of a sequence instead of its incompressibility. The Kolmogorov complexity characterizations and translation to Euclidean spaces are due to Mayordomo [24] and J. Lutz and Mayordomo [17]. Relationships between Hausdorff dimension and Kolmogorov complexity were also studied earlier by Ryabko [30, 31, 32], Staiger [33, 34], and Cai and Hartmanis [5]; see Section 6 of [15] for a detailed discussion of this history.

We will use the fact that these dimensions are preserved by sufficiently well-behaved functions, namely bi-Lipschitz computable bijections.

Lemma 3 (Reimann [28], Case and J. Lutz [6]). If $f: \mathbb{R}^{m} \rightarrow \mathbb{R}^{n}$ is computable and bi-Lipschitz, then $\operatorname{dim}(x)=\operatorname{dim}(f(x))$ and $\operatorname{Dim}(x)=\operatorname{Dim}(f(x))$ for all $x \in \mathbb{R}^{m}$.

\subsection{Conditional Dimensions}

The information theoretic nature of Definition 3.1 has led to the development of algorithmic dimensional quantities corresponding to the other algorithmic information theoretic quantities defined above. As analogues to mutual information and conditional information, Case and J. Lutz defined mutual dimensions [6], and J. Lutz and N. Lutz defined conditional dimensions. This work will use the latter, which we now describe.

Given $E \subseteq \mathbb{R}^{m}$ and $F \subseteq \mathbb{R}^{n}$, define

$$
K(E \mid F)=\max \left\{\min \left\{K(p \mid q): p \in E \cap \mathbb{Q}^{m}\right\}: q \in F \cap \mathbb{Q}^{n}\right\} .
$$

Then the conditional Kolmogorov complexity of $x \in \mathbb{R}^{m}$ at precision $r \in \mathbb{N}$ given $y \in \mathbb{R}^{n}$ at precision $s \in \mathbb{N}$ is given by

$$
K_{r, s}(x \mid y)=K\left(B_{2^{-r}}(x) \mid B_{2^{-s}}(y)\right) .
$$

- Definition 3.2 (J. Lutz and N. Lutz [16]). Let $x \in \mathbb{R}^{m}$ and $y \in \mathbb{R}^{n}$.

1. The lower conditional dimension of $x$ given $y$ is

$$
\operatorname{dim}(x: y)=\liminf _{r \rightarrow \infty} \frac{K_{r, r}(x \mid y)}{r} .
$$

2. The upper conditional dimension of $x$ given $y$ is

$$
\operatorname{Dim}(x: y)=\limsup _{r \rightarrow \infty} \frac{K_{r, r}(x \mid y)}{r} .
$$

That work also showed that the symmetry of algorithmic information holds in Euclidean space, in the form

$$
K_{r}(x, y)=K_{r}(x)+K_{r, r}(y \mid x)+o(r) .
$$

This fact and elementary properties of limits inferior and superior immediately imply the following chain rule for effective dimensions. 
- Theorem 4 (J. Lutz and N. Lutz [16]). For all $x \in \mathbb{R}^{m}$ and $y \in \mathbb{R}^{n}$,

$$
\begin{aligned}
\operatorname{dim}(x)+\operatorname{dim}(y \mid x) & \leq \operatorname{dim}(x, y) \\
& \leq \operatorname{dim}(x)+\operatorname{Dim}(y \mid x) \\
& \leq \operatorname{Dim}(x, y) \\
& \leq \operatorname{Dim}(x)+\operatorname{Dim}(y \mid x) .
\end{aligned}
$$

\subsection{Oracles and Relative Dimensions}

By making the fixed universal machine $U$ an oracle machine, the algorithmic information quantities above may be defined relative to any oracle $A \subseteq \mathbb{N}$. The definitions of $K^{A}(\sigma \mid \tau)$, $K^{A}(\sigma), K_{r}^{A}(x), K_{r}^{A}(x \mid y), \operatorname{dim}^{A}(x), \operatorname{Dim}^{A}(x), \operatorname{dim}^{A}(x \mid y)$ and $\operatorname{Dim}^{A}(x \mid y)$ all exactly mirror their unrelativized versions, except that $U$ is permitted to query membership in $A$ as a computational step.

For $y \in \mathbb{R}^{n}$, we write $\operatorname{dim}^{y}(x)$ as shorthand for $\operatorname{dim}^{A_{y}}(x)$, where $A_{y} \subseteq \mathbb{N}$ encodes the binary expansions of $y$ 's coordinates in some standard way, and similarly for $\operatorname{Dim}^{y}(x)$. Since this kind of oracle access to $y$ is at least as informative as any finite-precision estimate for $y$ (ignoring the small amount of information given by the precision parameter itself), these relative dimensions are bounded above by conditional dimensions.

- Lemma 5 (J. Lutz and N. Lutz [16]). For all $x \in \mathbb{R}^{m}$ and $y \in \mathbb{R}^{n}$,

1. $\operatorname{dim}^{y}(x) \leq \operatorname{dim}(x \mid y)$,

2. $\operatorname{Dim}^{y}(x) \leq \operatorname{Dim}(x \mid y)$.

\subsection{Point-to-Set Principle}

Effective Hausdorff dimension and effective packing dimension were conceived as constructive versions of classical Hausdorff dimension and packing dimension [15, 1]. The following point-to-set principle uses relativization to precisely characterize their relationships to their non-algorithmic precursors.

- Theorem 6 (J. Lutz and N. Lutz [16]). For every $E \subseteq \mathbb{R}^{n}$, the Hausdorff dimension and packing dimension of $E$ are

1. $\operatorname{dim}_{H}(E)=\min _{A \subseteq \mathbb{N}} \sup _{x \in E} \operatorname{dim}^{A}(x)$,

2. $\operatorname{dim}_{P}(E)=\min _{A \subseteq \mathbb{N}} \sup _{x \in E} \operatorname{Dim}^{A}(x)$.

Notice that, unlike the definitions of $\operatorname{dim}_{H}(E)$ and $\operatorname{dim}_{P}(E)$ given in Section 2, the above characterizations are completely symmetrical.

Theorem 6 allows us to prove lower bounds on classical dimensions in a pointwise way. To show a statement of the form $\operatorname{dim}_{H}(E) \geq \alpha$, it suffices to show, for a given oracle $A$ and every $\varepsilon>0$, that there exists an $x \in E$ satisfying $\operatorname{dim}^{A}(x) \geq \alpha-\varepsilon$. Unlike previous applications of this bounding technique [16, 18], the proofs in Sections 4 and 5 do not directly invoke Kolmogorov complexity; the only tools needed are Lemma 3, Theorem 4, Lemma 5, and Theorem 6 .

\section{$4 \quad$ Intersections of Fractals}

In this section we prove Theorem 1. We then use a symmetric argument to prove the corresponding statement for packing dimension, which is known [10]. For the case where 
$E, F \subseteq \mathbb{R}^{n}$ are Borel sets, Theorem 1 was shown in its present form by Falconer [11]. Closely related results, which also place restrictions on $E$ and $F$, were proven earlier by Mattila [21, 22] and Kahane [13].

- Theorem 1. For all $E, F \subseteq \mathbb{R}^{n}$, and for (Lebesgue) almost all $z \in \mathbb{R}^{n}$,

$$
\operatorname{dim}_{H}(E \cap(F+z)) \leq \max \left\{0, \operatorname{dim}_{H}(E \times F)-n\right\},
$$

where $F+z=\{x+z: x \in F\}$.

Proof. Let $E, F \subseteq \mathbb{R}^{n}$ and $z \in \mathbb{R}^{n}$. If $E \cap(F+z)=\emptyset$, then (1) holds trivially, so assume that the intersection is nonempty. Theorem 6 guarantees that there is some oracle set $A \subseteq \mathbb{N}$ satisfying

$$
\operatorname{dim}_{H}(E \times F)=\sup _{(x, y) \in E \times F} \operatorname{dim}^{A}(x, y) .
$$

It also guarantees, given any $\varepsilon>0$, that there is an $x \in E \cap(F+z)$ such that

$$
\operatorname{dim}^{A, z}(x) \geq \operatorname{dim}_{H}(E \cap(F+z))-\varepsilon .
$$

Since $(x, x-z) \in E \times F$, we have

$$
\begin{aligned}
\operatorname{dim}_{H}(E \times F) & \geq \operatorname{dim}^{A}(x, x-z) \\
& =\operatorname{dim}^{A}(x, z) \\
& \geq \operatorname{dim}^{A}(z)+\operatorname{dim}^{A}(x \mid z) \\
& \geq \operatorname{dim}^{A}(z)+\operatorname{dim}^{A, z}(x) \\
& \geq \operatorname{dim}^{A}(z)+\operatorname{dim}_{H}(E \cap(F+z))-\varepsilon .
\end{aligned}
$$

The above lines follow from (2), Lemma 3, Theorem 4, Lemma 5, and (3), respectively. Letting $\varepsilon \rightarrow 0$, we have

$$
\operatorname{dim}_{H}(E \cap(F+z)) \leq \operatorname{dim}_{H}(E \times F)-\operatorname{dim}^{A}(z) .
$$

Thus, (a) holds whenever $\operatorname{dim}^{A}(z)=n$. In particular, it holds when $z$ is Martin-Löf random relative to $A$, i.e., for Lebesgue almost all $z \in \mathbb{R}^{n}[14,20]$.

For the case that $E$ and $F$ are Borel sets, Falconer [11] notes that the intersection formula is readily extended to rigid motions and similarities. The same argument applies in the general case, so Theorem 1 has the following corollary.

- Corollary 7. Let $E, F \subseteq \mathbb{R}^{n}$. Let $G$ be the group of rigid motions or the group of similarities on $\mathbb{R}^{n}$. Then, for almost all $\sigma \in G$,

$$
\operatorname{dim}_{H}(E \cap \sigma(F)) \leq \max \left\{0, \operatorname{dim}_{H}(E \times F)-n\right\} .
$$

Proof (Following Falconer [11]). For all rotations (and all scalings) of $F$, Theorem 1 tells us that (4) holds for almost all translations. Thus, (4) holds for almost all rigid motions and almost all similarities.

A corresponding intersection formula for packing dimension has been shown for arbitrary $E, F \subseteq \mathbb{R}^{n}$ by Falconer [10]. That proof is not difficult or long, but an algorithmic dimensional proof is presented here as an instance where this technique applies symmetrically to both Hausdorff and packing dimension. 
- Theorem 8 (Falconer [10]). For all $E, F \subseteq \mathbb{R}^{n}$, and for (Lebesgue) almost all $z \in \mathbb{R}^{n}$, $\operatorname{dim}_{P}(E \cap(F+z)) \leq \max \left\{0, \operatorname{dim}_{P}(E \times F)-n\right\}$.

Proof. As in Theorem 1, we may assume that the intersection is nonempty. Apply Theorem 6 to choose an oracle set $B \subseteq \mathbb{N}$ such that

$$
\operatorname{dim}_{P}(E \times F)=\sup _{(x, y) \in E \times F} \operatorname{Dim}^{B}(x, y)
$$

and, given $\varepsilon>0$, a point $y \in E \cap(F+z)$ satisfying

$$
\operatorname{Dim}^{B, z}(y) \geq \operatorname{dim}_{P}(E \cap(F+z))-\varepsilon .
$$

Then $(y, y-z) \in E \times F$, and we may proceed much as before:

$$
\begin{aligned}
\operatorname{dim}_{P}(E \times F) & \geq \operatorname{Dim}^{B}(y, y-z) \\
& =\operatorname{Dim}^{B}(y, z) \\
& \geq \operatorname{dim}^{B}(z)+\operatorname{Dim}^{B}(y \mid z) \\
& \geq \operatorname{dim}^{B}(z)+\operatorname{Dim}^{B, z}(y) \\
& \geq \operatorname{dim}^{B}(z)+\operatorname{dim}_{P}(E \cap(F+z))-\varepsilon .
\end{aligned}
$$

These lines follow from (5), Lemma 3, Theorem 4, Lemma 5, and (6). Again, $\operatorname{dim}^{B}(z)=n$ for almost every $z \in \mathbb{R}^{n}$, so this completes the proof.

\section{Products of Fractals}

In this section we prove four known product inequalities for fractal dimensions. Inequality (7), which was stated in the introduction as Theorem 2, is due to Marstrand [19]. When $E$ and $F$ are Borel sets, it is simple to prove (7) by using Frostman's Lemma, but the argument for general sets using net measures is considerably more difficult [23, 9]. The other three inequalities are due to Tricot [37]. Reference [23] gives a more detailed account of this history.

- Theorem 9 (Marstrand [19], Tricot [37]). For all $E \subseteq \mathbb{R}^{m}$ and $F \subseteq \mathbb{R}^{n}$,

$$
\begin{aligned}
\operatorname{dim}_{H}(E)+\operatorname{dim}_{H}(F) & \leq \operatorname{dim}_{H}(E \times F) \\
& \leq \operatorname{dim}_{H}(E)+\operatorname{dim}_{P}(F) \\
& \leq \operatorname{dim}_{P}(E \times F) \\
& \leq \operatorname{dim}_{P}(E)+\operatorname{dim}_{P}(F) .
\end{aligned}
$$

Notice the superficial resemblance of this theorem to Theorem 4 . This similarity is not a coincidence; each inequality in Theorem 9 follows from the corresponding line in Theorem 4. The arguments given here for (7-10) are each similar in length to the proof of (7) for Borel sets. That is, they are quite short.

Proof. Theorem 6 guarantees, for every $\varepsilon>0$, that there exist an oracle set $A \subseteq \mathbb{N}$ and points $x \in E$ and $y \in F$ such that

$$
\begin{aligned}
\operatorname{dim}_{H}(E \times F) & =\sup _{z \in E \times F} \operatorname{dim}^{A}(z), \\
\operatorname{dim}^{A}(x) & \geq \operatorname{dim}_{H}(E)-\varepsilon, \\
\operatorname{dim}^{A, x}(y) & \geq \operatorname{dim}_{H}(F)-\varepsilon .
\end{aligned}
$$


Then by (11), Theorem 4 relative to $A$, and Lemma 5 relative to $A$, we have

$$
\begin{aligned}
\operatorname{dim}_{H}(E \times F) & \geq \operatorname{dim}^{A}(x, y) \\
& \geq \operatorname{dim}^{A}(x)+\operatorname{dim}^{A}(y \mid x) \\
& \geq \operatorname{dim}^{A}(x)+\operatorname{dim}^{A, x}(y) \\
& \geq \operatorname{dim}_{H}(E)+\operatorname{dim}_{H}(F)-2 \varepsilon,
\end{aligned}
$$

by our choice of $x$ and $y$. Since $\varepsilon>0$ was arbitrary, we conclude that (7) holds.

For (8), let $\varepsilon>0$ and use both parts of Theorem 6 to find $B, C \subseteq \mathbb{N}, u \in E$, and $v \in F$ such that

$$
\begin{aligned}
\operatorname{dim}_{H}(E) & =\sup _{x \in E} \operatorname{dim}^{B}(x), \\
\operatorname{dim}_{P}(F) & =\sup _{y \in E} \operatorname{Dim}^{C}(y), \\
\operatorname{dim}^{B, C}(u, v) & \geq \operatorname{dim}_{H}(E \times F)-\varepsilon .
\end{aligned}
$$

Since $B$ and $C$ minimize their respective expressions, we also have

$$
\begin{aligned}
\operatorname{dim}_{H}(E) & =\sup _{x \in E} \operatorname{dim}^{B, C}(x), \\
\operatorname{dim}_{P}(F) & =\sup _{y \in E} \operatorname{Dim}^{B, C}(y) .
\end{aligned}
$$

Thus, we can apply Theorem 4 relative to $B, C$, after first noticing that conditioning on another point never increases dimension.

$$
\begin{aligned}
\operatorname{dim}_{H}(E)+\operatorname{dim}_{P}(F) & \geq \operatorname{dim}^{B, C}(u)+\operatorname{Dim}^{B, C}(v) \\
& \geq \operatorname{dim}^{B, C}(u \mid v)+\operatorname{Dim}^{B, C}(v) \\
& \geq \operatorname{dim}^{B, C}(u, v) \\
& \geq \operatorname{dim}_{H}(E \times F)-\varepsilon .
\end{aligned}
$$

Again, $\varepsilon$ was arbitrary, so (8) holds.

For (9) and (10), we use essentially the same arguments as above. By Theorem 6, there are $A^{\prime}, B^{\prime} \subseteq \mathbb{N}, x^{\prime}, u^{\prime} \in E, y^{\prime}, v^{\prime} \in F$, and $\varepsilon>0$ that satisfy

$$
\begin{aligned}
\operatorname{dim}_{P}(E \times F) & =\sup _{z \in E \times F} \operatorname{Dim}^{A^{\prime}}(z), \\
\operatorname{dim}_{H}(E) & =\sup _{z \in E} \operatorname{Dim}^{B^{\prime}}(z), \\
\operatorname{dim}^{A^{\prime}}\left(x^{\prime}\right) & \geq \operatorname{dim}_{H}(E)-\varepsilon, \\
\operatorname{Dim}^{A^{\prime}, x^{\prime}}\left(y^{\prime}\right) & \geq \operatorname{dim}_{P}(F)-\varepsilon, \\
\operatorname{Dim}^{B^{\prime}, C}\left(u^{\prime}, v^{\prime}\right) & \geq \operatorname{dim}_{P}(E \times F)-\varepsilon,
\end{aligned}
$$

where $x$ and $C$ are as above. We once again apply relativized versions of Theorem 4 and 
Lemma 5:

$$
\begin{aligned}
\operatorname{dim}_{P}(E)+\operatorname{dim}_{P}(F) & \geq \operatorname{Dim}^{B^{\prime}, C}\left(u^{\prime}\right)+\operatorname{Dim}^{B^{\prime}, C}\left(v^{\prime}\right) \\
& \geq \operatorname{Dim}^{B^{\prime}, C}\left(u^{\prime} \mid v^{\prime}\right)+\operatorname{Dim}^{B^{\prime}, C}\left(v^{\prime}\right) \\
& \geq \operatorname{Dim}^{B^{\prime}, C}\left(u^{\prime}, v^{\prime}\right) \\
& \geq \operatorname{dim}_{P}(E \times F)-\varepsilon \\
& \geq \operatorname{Dim}^{A^{\prime}}\left(x^{\prime}, y^{\prime}\right)-\varepsilon \\
& \geq \operatorname{dim}^{A^{\prime}}\left(x^{\prime}\right)+\operatorname{Dim}^{A^{\prime}}\left(y^{\prime} \mid x^{\prime}\right)-\varepsilon \\
& \geq \operatorname{dim}^{A^{\prime}}\left(x^{\prime}\right)+\operatorname{Dim}^{A^{\prime}, x^{\prime}}\left(y^{\prime}\right)-\varepsilon \\
& \geq \operatorname{dim}_{H}(E)+\operatorname{dim}_{P}(F)-3 \varepsilon
\end{aligned}
$$

Letting $\varepsilon \rightarrow 0$ completes the proof.

\section{Conclusion}

The applications of theoretical computer science to pure mathematics in this paper yielded a significant extension to a basic theorem on Hausdorff dimension, as well as a much simpler argument for another such theorem. Understanding classical fractal dimensions as pointwise, algorithmic information theoretic quantities enables reasoning about them in a way that is both fine-grained and intuitive, and the proofs in this work are further evidence of the power and versatility of bounding techniques using Theorem 6 . In particular, Theorem 1 demonstrates that this approach can be used to strengthen the foundations of fractal geometry. Therefore, in addition to further applications of these techniques, developing more refined results on the relationship between classical geometric measure theory and Kolmogorov complexity is an appealing direction for future investigations.

\section{References}

1 Krishna B. Athreya, John M. Hitchcock, Jack H. Lutz, and Elvira Mayordomo. Effective strong dimension in algorithmic information and computational complexity. SIAM Journal of Computing, 37(3):671-705, 2007. doi:10.1137/s0097539703446912.

2 Verónica Becher, Jan Reimann, and Theodore A. Slaman. Irrationality exponent, Hausdorff dimension and effectivization. arXiv:1601.00153 [math.NT], 2016.

3 Christopher J. Bishop. Personal communication, April 27, 2017.

4 Christopher J. Bishop and Yuval Peres. Fractals in Probability and Analysis. Cambridge University Press, 2017. doi:10.1017/9781316460238.

5 Jin-Yi Cai and Juris Hartmanis. On Hausdorff and topological dimensions of the Kolmogorov complexity of the real line. Journal of Computer and System Sciences, 49(3):605619, 1994. doi:10.1016/S0022-0000(05)80073-X.

6 Adam Case and Jack H. Lutz. Mutual dimension. ACM Transactions on Computation Theory, 7(3):12, 2015. doi:10.1145/2786566.

7 R. O. Davies. Some remarks on the Kakeya problem. Proceedings of the Cambridge Philosophical Society, 69:417-421, 1971. doi:10.1017/s0305004100046867.

8 Rod Downey and Denis Hirschfeldt. Algorithmic Randomness and Complexity. SpringerVerlag, 2010. doi:10.1007/978-0-387-68441-3.

9 Kenneth J. Falconer. The Geometry of Fractal Sets. Cambridge University Press, 1985. doi: $10.1017 /$ cbo9780511623738. 
10 Kenneth J. Falconer. Sets with large intersection properties. Journal of the London Mathematical Society, 49(2):267-280, 1994. doi:10.1112/jlms/49.2.267.

11 Kenneth J. Falconer. Fractal Geometry: Mathematical Foundations and Applications. Wiley, third edition, 2014. doi:10.1002/0470013850.

12 Felix Hausdorff. Dimension und äusseres Mass. Mathematische Annalen, 79:157-179, 1919. doi: 10.1007/978-3-642-59483-0_2.

13 Jean-Pierre Kahane. Sur la dimension des intersections. In Jorge Alberto Barroso, editor, Aspects of mathematics and its applications, North-Holland Mathematical Library, 34, pages 419-430. Elsevier, 1986. doi:10.1016/s0924-6509(09)70272-7.

14 Ming Li and Paul M.B. Vitányi. An Introduction to Kolmogorov Complexity and Its Applications. Springer, third edition, 2008. doi:10.1007/978-0-387-49820-1.

15 Jack H. Lutz. The dimensions of individual strings and sequences. Information and Computation, 187(1):49-79, 2003. doi:10.1016/s0890-5401(03)00187-1.

16 Jack H. Lutz and Neil Lutz. Algorithmic information, plane Kakeya sets, and conditional dimension. In Proceedings of the 34th Symposium on Theoretical Aspects of Computer Science, STACS 2017, March 8-11, 2017, Hannover, Germany, pages 53:1-53:13, 2017. doi: 10.4230/LIPIcs.STACS. 2017.53.

17 Jack H. Lutz and Elvira Mayordomo. Dimensions of points in self-similar fractals. SIAM Journal of Computing, 38(3):1080-1112, 2008. doi:10.1007/978-3-540-69733-6_22.

18 Neil Lutz and D. M. Stull. Bounding the dimension of points on a line. In TV Gopal, Gerhard Jaeger, and Silvia Steila, editors, Theory and Applications of Models of Computation: 14th Annual Conference, TAMC 2017, Bern, Switzerland, April 20-22, 2017, Proceedings, pages 425-439, 2017. doi:10.1007/978-3-319-55911-7_31.

19 John M. Marstrand. Some fundamental geometrical properties of plane sets of fractional dimensions. Proceedings of the London Mathematical Society, 4(3):257-302, 1954 . doi: 10.1112/plms/s3-4.1.257.

20 Per Martin-Löf. The definition of random sequences. Information and Control, 9(6):602619, 1966. doi:10.1016/s0019-9958(66)80018-9.

21 Pertti Mattila. Hausdorff dimension and capacities of intersections of sets in $n$-space. Acta Mathematica, 152:77-105, 1984. doi:10.1007/bf02392192.

22 Pertti Mattila. On the Hausdorff dimension and capacities of intersections. Mathematika, 32:213-217, 1985. doi:10.1112/s0025579300011001.

23 Pertti Mattila. Geometry of sets and measures in Euclidean spaces: fractals and rectifiability. Cambridge University Press, 1995. doi:10.1017/cbo9780511623813.

24 Elvira Mayordomo. A Kolmogorov complexity characterization of constructive Hausdorff dimension. Inf. Process. Lett., 84(1):1-3, 2002. doi:10.1016/s0020-0190(02)00343-5.

25 Elvira Mayordomo. Effective fractal dimension in algorithmic information theory. In S. Barry Cooper, Benedikt Löwe, and Andrea Sorbi, editors, New Computational Paradigms: Changing Conceptions of What is Computable, pages 259-285. Springer New York, 2008. doi:10.1007/978-0-387-68546-5_12.

26 Ursula Molter and Ezequiel Rela. Furstenberg sets for a fractal set of directions. Proceedings of the American Mathematical Society, 140:2753-2765, 2012. doi:10.1090/ s0002-9939-2011-11111-0.

27 Andre Nies. Computability and Randomness. Oxford University Press, Inc., New York, NY, USA, 2009. doi:10.1093/acprof : oso/9780199230761.001.0001.

28 Jan Reimann. Computability and fractal dimension. PhD thesis, Heidelberg University, 2004.

29 Jan Reimann. Effectively closed sets of measures and randomness. Annals of Pure and Applied Logic, 156(1):170-182, 2008. doi:10.1016/j .apal.2008.06.015. 
30 Boris Ryabko. Noiseless coding of combinatorial sources. Problems of Information Transmission, 22:170-179, 1986.

31 Boris Ryabko. Algorithmic approach to the prediction problem. Problems of Information Transmission, 29:186-193, 1993.

32 Boris Ryabko. The complexity and effectiveness of prediction algorithms. Journal of Complexity, 10(3):281-295, 1994. doi:10.1006/jcom.1994.1015.

33 Ludwig Staiger. Kolmogorov complexity and Hausdorff dimension. Information and Computation, 103:159-194, 1989. doi:10.1007/3-540-51498-8_42.

34 Ludwig Staiger. A tight upper bound on Kolmogorov complexity and uniformly optimal prediction. Theory of Computing Systems, 31:215-229, 1998. doi:10.1007/s002240000086.

35 Elias M. Stein and Rami Shakarchi. Real Analysis: Measure Theory, Integration, and Hilbert Spaces. Princeton Lectures in Analysis. Princeton University Press, 2005.

36 Dennis Sullivan. Entropy, Hausdorff measures old and new, and limit sets of geometrically finite Kleinian groups. Acta Mathematica, 153(1):259-277, 1984. doi:10.1007/bf02392379.

37 Claude Tricot. Two definitions of fractional dimension. Mathematical Proceedings of the Cambridge Philosophical Society, 91(1):57-74, 1982. doi:10.1017/s0305004100059119. 